\title{
Causas de cosechado y características de hembras bovinas (Bos taurus, Bos indicus, Artyodactila: Bovidae) cosechadas en planta en Costa Rica del 2008 al 2009
}

\author{
Roger Molina Coto, Carlos Arroyo Oquendo, José Ramón Molina Villalobos \& Rodolfo WingChing-Jones
}

Escuela de Zootecnia, Facultad de Ciencias Agroalimentarias, Universidad de Costa Rica, San Pedro de Montes de Oca, San José, Costa Rica; roger.molina@ucr.ac.cr, carlos.arroyo@ucr.ac.cr jose.molinavillalobos@ucr.ac.cr, rodolfo.wingching@ucr.ac.cr

Recibido 1-X-2013 • Corregido 11-XII-2013 • Aceptado 30-I-2014

\begin{abstract}
Reasons for culling and number of bovine females (Bos taurus, Artyodactila: Bovidae) culled in plant in Costa Rica from 2008 through 2009. It is important for the livestock sector to keep regular checks on production, reproduction and industrialization to prevent any changes that may affect it, and use the information as a basis for decision-making. The main purpose of this job was to define the quantity and main causes for culling bovine females in one of the most important slaughterhouse in Costa Rica. From October 2008 to December 2009 were quantified the bovine females sent to Cooperativa Matadero Nacional de Montecillos R.L., Alajuela, Costa Rica; this coop represents up to $20 \%$ of the national herd slaughtered. During the period of the study, the percentage of cows culled was $50.57 \%$, from this, $51.44 \%$ were pregnant in stages from conceptuses to fetuses in the last days before calving. There is a $38.16 \%$ of young bovine females that are harvested, some of them without the chance for calving. Among people associated to the Coop the causes for harvesting cows are: old cows (19.6\%), selection pressure (18.2\%), lack of money (16.1\%), reproductive issues (16.1\%), shortage of feed for the animals (18.2\%) and others like sicknesses and market strategies (16.0\%). Some of these causes can be solved if farms are administered correctly avoiding losses for farmers and the decrease of the national herd.
\end{abstract}

KEY WORDS: Pregnant cows, bovine herd, harvest of animals, extractions, gestation.

\section{RESUMEN}

Es importante para el sector ganadero mantener controles periódicos sobre producción, reproducción e industrialización para prever cualquier cambio que pueda afectarlo, y utilizar la información como base para la toma decisiones. El estudio se realizó desde octubre 2008 a diciembre de 2009 en la Cooperativa Matadero Nacional de Montecillos R.L, Alajuela, Costa Rica; donde se cuantificó la cantidad de hembras bovinas cosechadas; dicha cooperativa representa más del $20 \%$ de las extracciones bovinas del país. Durante el periodo, el porcentaje de hembras que se sacrificó fue de 50,57\%, 51,44\% fueron hembras en estado de preñez, desde gestaciones tempranas donde apenas se trataba de capsulas embrionarias hasta fetos en avanzados estados de desarrollo. Se encontró que un $38,16 \%$ de hembras que se sacrifican en edades tempranas, algunas no llegaron a tener actividad reproductiva. Entre las razones de cosechar hembras, según la opinión de los socios de la Cooperativa Montecillos, se determinaron el animal viejo (19,6\%), presión de selección $(18,2 \%)$, necesidad de dinero $(16,1 \%)$, problemas de reproducción (16,1\%), escasez de alimento para los animales $(14,0 \%)$ y otras como enfermedades y estrategia de mercado (16,0\%). Algunas de las causas se pueden resolver con una eficiente administración de las fincas para evitar pérdidas para el ganadero y para el hato nacional.

PALABRAS CLAVE: Hembras preñadas, hato bovino, sacrificio de animales, extracciones, gestación.
El sector ganadero costarricense debe mantener controles periódicos sobre producción, reproducción e industrialización de los sistemas nacionales, que le permitan contar con información actualizada para prever cualquier cambio que pueda afectarlo, y utilizar la información como base para la toma decisiones (Ugalde, 1996).

Los censos ganaderos y encuestas realizadas en el país, exponen el comportamiento o tendencias de los sistemas ganaderos costarricenses (CORFOGA, 2000). En el ambiente ganadero costarricense se habla del sacrificio de hembras bovinas en el hato nacional, entidades como el Consejo Nacional de Producción (CNP) y la Corporación de Fomento Ganadera (CORFOGA) publican desde el año 2000 y 2003 respectivamente este tipo de información, en formatos que no siempre son accesibles a los productores ganaderos o público en general. La cosecha de hembras bovinas no puede ser tan sólo parte de 
una estadística, sin profundizar en la repercusión sobre la sostenibilidad del hato nacional, puesto que ésta práctica se considera un paso atrás en los esfuerzos de mejoramiento genético, programas de manejo nutricional y de mejora de los sistemas productivos (Khan \& Khan, 1989). Por su parte, Oses (2002) informa que la posición de CORFOGA, es disminuir las tasas de extracción de semovientes, hembras de carne y doble propósito, así como mejorar los parámetros reproductivos (Intervalo entre partos, Edad a Primer Parto, Periodo Abierto) en el hato.

Por ejemplo, en el año 1982, el hato costarricense estaba compuesto por 2276000 cabezas de las cuáles 1531 000 correspondía a hembras, mientras que para el año 2000 fue de 1358000 cabezas con 956000 hembras, lo que indica una reducción del $40 \%$ del hato general y del $38 \%$ de las hembras (Pérez, s.f.). Acerca de la cantidad de hembras preñadas que se extraen o cosechan del hato en Costa Rica existe poca información. A nivel costarricense, existen dos informes sobre este tema, en el año 1982 en el Matadero Municipal de Cerrillos de Cartago, donde en un periodo de dos meses se registró la cosecha de 1000 hembras preñadas (Muller, 1982), mientras que, en el año 1985 en la Cooperativa Matadero Nacional de Montecillos (Coopemontecillos R.L.) durante tres meses, se obtuvo la cosecha de 7309 hembras preñadas (Morales, 1985).

De forma paralela el análisis de la información sobre sacrificio de hembras bovinas, se debe integrar con la evaluación de índices reproductivos y productivos del país, por ejemplo Méndez (2008), determinó un 53\% de parición en la región Chorotega, lo que combinado a una alta tasa de extracción de hembras en esa zona, reduce la capacidad del hato de incrementarse.

El estudio de las causas por las cuáles el ganadero sacrifica hembras y entre las mismas, hembras preñadas, merece un análisis que permita identificar el porqué de este tipo de decisión, para determinar las acciones necesarias que mitigue una eventual disminución del hato costarricense y las pérdidas económicas para los ganaderos. Por tal motivo, el objetivo de este trabajo fue determinar la cantidad de hembras que se industrializan en una planta de cosecha nacional en un periodo de tiempo y citar las razones por las cuáles, los dueños de estos semovientes, destinan a cosecha estos animales.

\section{METODOLOGÍA}

Ubicación de la evaluación. En la planta de cosecha de la Cooperativa Matadero Nacional de Montecillos (Coopemontecillos R.L.), ubicada en Alajuela, provincia número dos de Costa Rica, durante 14 meses comprendidos entre octubre 2008 y diciembre 2009, se realizó una estimación en la cantidad de semovientes hembras que se destinan para cosecha. Además, se cuantificó la cantidad de hembras preñadas que se cosecharon durante ese mismo periodo.

Toma de la información. Para la estimación de la cantidad de hembras que se cosechan, se realizó una visita semanal variando consecutivamente el día de la semana, durante el periodo de investigación, el conteo se realizó de manera alterna en la misma visita en dos zonas, a nivel de la planta de cosecha y en la zona de "rendering" (zona de molido y secado de remanentes de planta), debido a que durante el periodo de investigación, se identificó dos tipos de poblaciones de hembras según la etapa de la gestación. Los fetos a nivel de la planta se observaron de forma directa y eran los de mayor tamaño, mientras que, en el sector de "rendering" se realizó la exploración de los úteros por medio de la exposición de su interior a través de un bisturí. Se evaluaron todas las hembras cosechadas sin discriminar si pertenecían a la especie Bos taurus, Bos índicus o cualquiera de las razas bovinas y sus cruces. Todos los días en los que se contabilizaron las preñeces, se recopilo la información desde el inicio de labores en la planta de cosecha hasta el final de la operación de ese día. El conteo incluyó animales provenientes de diferentes regiones del país, edades, patrón racial y de productores asociados de la cooperativa y clientes que compran el servicio de la planta. También se usó información sobre la edad dentaria de las hembras sacrificadas en cada día de muestreo, ésta información fue tomada por la planta al valorar la presencia de dientes deciduos o permanentes y su desgaste en la mandíbula de los animales. La planta también facilitó sus registros de la cantidad de machos, hembras y terneros, clasificados de acuerdo a la proveniencia de asociados o clientes independientes.

Encuesta. Con relación a las causas que llevan a los dueños de los semovientes a cosechar hembras vacías o preñadas, se realizó una encuesta telefónica a 175 asociados de la Cooperativa Matadero Nacional de Montecillos, en ésta se recopilaron datos provenientes de todo el país de ganaderos que entregaron hembras durante el periodo experimental con el objetivo de conocer las causas por las cuáles cosecharon hembras preñadas. Esta encuesta, consideró, si se trataba de criadores, intermediarios y que tipo de controles tenía en su finca como registros y palpaciones. 
Análisis de la información. La información recopilada se tabuló en una hoja electrónica de Excel 2010 de Microsoft Office ${ }^{\oplus}$, en donde los datos se organizaron según la fecha del muestreo, lugar de muestreo y condición (gestante o no gestante). Por medio de medidas de tendencia central, la información fue analizada (Quintana, 1996). En el caso, de la recopilación de datos por vía telefónica, se organizó la información en una hoja electrónica, en donde se consideró el asociado, la localidad y se contabilizó la causa que motiva al productor a cosechar los semovientes, cuando se registró más de una causa, esta se priorizó y se analizó la información de manera porcentual con relación al total de entrevistas.

\section{RESULTADOS}

En la figura 1 se observa el comportamiento en la cantidad de machos y hembras sacrificados en la planta a lo largo del periodo de muestreo, así como la cantidad absoluta y porcentual de hembras preñadas. Se observa que el porcentaje promedio de hembras que se cosecharon fue de 50,57\%, lo que significa más de 1856 hembras por mes, de éstas el $51,40 \% \pm 9,09 \%$ fueron hembras preñadas.

Según los datos de cosecha provenientes de la planta, el $64,86 \%$ del total de hembras que se industrializaron provienen de los asociados de la Cooperativa, el porcentaje restante de la venta de servicios. En este sentido, cerca del $91 \%$ de los productores entregaron hembras para su cosecha y más del 63\% fueron novillas.

El análisis de la edad dentaria de los animales cosechados, indicó que el 38,16\% de hembras cosechadas presentan menos de ocho dientes permanentes, lo que significa un animal con una edad fisiológica igual o menor a los 40 meses (Rodríguez, 2008 comm pers.).

En cuanto a las razones de cosecha de hembras, las principales causas por las cuales el productor comercializa hembras para su sacrificio fueron considerar al animal una vaca vieja $(19,6 \%)$, la presión de selección de los animales $(18,2 \%)$, necesidad de dinero $(16,1 \%)$, problemas de reproducción $(16,1 \%)$, escasez de alimento para los animales $(14,0 \%)$ y otras razones minoritarias como enfermedades o estrategia de mercado $(16,0 \%)$. Como resultado del análisis de la información solicitada en la encuesta, se determinó que la carencia de registros, que permiten un control reproductivo de los semovientes y la trazabilidad de los mismos agudiza la extracción de hembras del sistema, por desconocimiento.

\section{DISCUSIÓN}

El periodo experimental coincidió con una de las crisis más agudas de la ganadería de los últimos tiempos (Pérez, 2010), esto provocó que no sólo aumentara el número total de animales cosechados sino también el número de hembras aumentó motivado por los precios bajos del mercado. Del año 2000 al 2007, se cosecharon menos de 400000 cabezas de ganado por año en el país, mientras que en el 2008 fueron 401750 y en el 2009 fue de 438639 (CNP, 2010).

Al comparar los resultados de la presente investigación con la información recopilada en el Cuadro 1, se pueden hacer dos comparaciones generales, la primera a nivel exterior donde se observa que Costa Rica ocupa los primeros lugares de cosecha de hembras bovinas y de porcentajes altos en cuanto a la cosecha de hembras

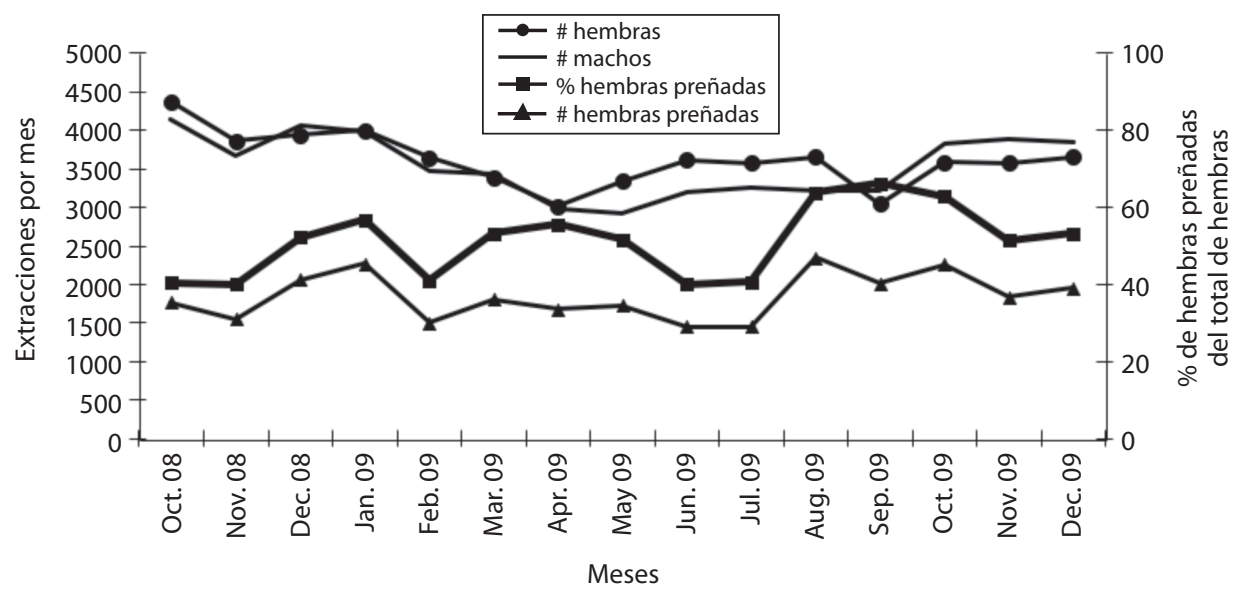

Fig. 1. Extracción de machos, hembras totales y preñadas y porcentaje de hembra preñada desde octubre de 2008 a diciembre de 2009 en Coopemontecillos; Alajuela, Costa Rica (2009). 
CUADRO 1

Los porcentajes de hembras totales y hembras preñadas determinados en diferentes países.

\begin{tabular}{lcccl} 
País & \% Hembras & $\begin{array}{c}\text { \% Hembras } \\
\text { preñadas }\end{array}$ & $\begin{array}{c}\text { Duración } \\
\text { del estudio }\end{array}$ & Fuente \\
Costa Rica & 88,0 & 60,1 & 2 meses & Muller, 1982 \\
Nicaragua & 64,0 & 60,0 & 3 meses & Morales, 1985 \\
México & 13,6 & 34,3 & 6 meses & Centeno y Marenco, 2007 \\
& - & 53,6 & - & Vázquez et al., 1993 \\
Camerún & - & 66,3 & - & Erales, Ortega, Rodríguez y Segura, 2008 \\
Etiopía & 35,0 & 22,1 & - & Ndi, Tambi y Agharid, 1993 \\
Nigeria & - & 28,9 & - & Simenew, Bekana, Fikre, Tilahum y Wondú, 2011 \\
Pakistán & 62,0 & 20,0 & 3 meses & Adama, Shiawoya, y Michael, 2011 \\
Irán & - & 8,6 & - & Khan y Khan, 1989 \\
\hline
\end{tabular}

preñadas se refiere y la otra a nivel interno, donde a pesar de que se generaron datos más favorables que en las investigaciones previas, se debe tomar en cuenta la longitud del periodo de experimentación y en el caso de Muller (1982), éste justifica su resultado por la ubicación de la planta, la cual estaba rodeada de sistemas de producción lácteos, por lo tanto, la mayoría de los semovientes que se cosechaban presentan un patrón de vaca lechera de desecho. Es destacable además que durante el periodo de ejecución de este estudio, ésta planta de cosecha representó el $20,73 \%$ de la cosecha bovina costarricense (CNP, 2010), siendo una de las cuatro plantas principales de Costa Rica en las cuáles se cosecha entre el 80 y $90 \%$ de las extracciones totales del país (Holmann, Rivas, Pérez, Castro, Schuetz, \& Rodríguez, 2007; MEIC, 2009).

La información sobre el tema de cosecha de hembras preñadas no es muy accesible, lo cual eventualmente, podría llevar a tener que tomar medidas paliativas que no favorecen el desarrollo de la ganadería. En el año 2007 en Colombia, después de detectar una marcada disminución del hato bovino, el gobierno, por medio del Ministerio de Agricultura y Desarrollo Rural de ese país, emitió una resolución en la que prohibió el sacrificio de hembras bovinas aptas para la reproducción y definió como hembras aptas para la reproducción, a aquellas que poseen un correcto estado sanitario, en edad reproductiva y libre de problemas de infertilidad (Comvezcol, 2007). Esta resolución deja de lado el descarte de hembras por motivos de producción y además provoca disminución en la selección de animales, esto permite que la mejora genética retroceda (Comvezcol 2007). En el caso de Argentina, la cosecha de hembras, provocó que el hato nacional se redujera en 8 millones de cabezas, producto de la extracción de más del $50 \%$ de hembras (Crudeli, 2010). Con similares consecuencias, la cosecha de hembras en Nicaragua, provocó que se dejara de percibir 426.200 dólares, por concepto del valor de las crías al destete, sin contemplar las pérdidas en hembras reproductoras (Centeno \& Marenco, 2007).

En el caso de la evaluación de la edad fisiológica de las hembras cosechadas por medio de la dentición, un porcentaje considerable son hembras que si cuentan con las condiciones sanitarias, genéticas y de fertilidad adecuadas, son animales aptos para la reproducción, según Hafez (1996), para que la hembra bovina se reproduzca con éxito debe haber alcanzado la madurez sexual que viene después de la pubertad, la pubertad es alcanzada entre los 10 y 12 meses en las hembras de razas lecheras, entre 11 y 15 meses en ganado de engorde Bos taurus y entre 18 y 24 meses para razas cebuínas. Por lo anterior, el porcentaje de hembras menores a 8 dientes que son cosechadas, son hembras en edad productiva.

Por otro lado, se determinaron las razones por las cuáles se cosechan hembras como tales y hembras gestantes, algunos de los resultados coinciden con los informados por Simenew et al. (2011) en donde el descarte de hembras en Etiopía se da por infertilidad (33\%), edad (15\%) y necesidades económicas (13,3\%).

La época en la que se desarrolló este estudio fue una coyuntura especial de los precios del ganado bovino, pero genera una información para que los entes rectores de la actividad ganadera controlen la cosecha de hembras del hato para evitar repercusiones negativas en la evolución del hato debido a ésta práctica. Además, se refleja un contraste entre los indicadores reproductivos del hato nacional y el desperdicio real de potenciales crías de hembras que se sacrifican preñadas. 
La experiencia en otros países establece un conflicto entre la mejora genética y el crecimiento del hato, debido a que la extracción de hembras produce un efecto negativo en el hato total, pero su control provoca una posterior retención de hembras con características menos ideales para la ganadería.

Los ganaderos deben acoger tecnologías que les permitan producir de manera eficiente, sin pensar únicamente en indicadores productivos como ganancias de peso o kilogramos de leche producida, sino mejorar los índices reproductivos. El uso de identificación y registros de los animales, el control reproductivo, los cruzamientos dirigidos, la planificación de la finca y la producción y el seguimiento de objetivos claros, son parte de las opciones que tienen muchos ganaderos para mejorar su producción, mitigar los efectos de las crisis y en conjunto favorecer el sector ganadero en Costa Rica.

\section{AGRADECIMIENTOS}

Se agradece a los funcionarios de Coopemontecillos que autorizaron este trabajo y que facilitaron la información de la planta, a los trabajadores de planta que colaboraron en el proceso de recolección de datos y a los asociados que atendieron la encuesta telefónica.

\section{REFERENCIAS}

Adama J, Shiawoya E., \& Michael N. (2011). Incidence of foetal wastages of cows slaughtered in Minna abattoir, Niger state, Nigeria. Journal of Applied Biosciences, 42, 2876 $-2881$

CORFOGA. (2000). Censo Ganadero. Corporación de Fomento Ganadero. San José, Costa Rica. Recuperado de: www. mag.go.cr/biblioteca_virtual_animal/censo-ganadero-2000.pdf

Centeno, A., \& Marenco, B. (2007). Estudio descriptivo de las hembras bovinas gestantes sacrificadas en el Matadero Central S.A; MACESA (Tesis de licenciatura). Universidad Nacional Agraria. Juigalpa, Nicaragua.

Consejo Nacional de la Producción (CNP). Información del mercado bovino. 2010. San José, Costa Rica. Recuperado de: http://www.cnp.go.cr/index.php

Comvezcol. (2007). Consejo de Médicos Veterinarios Zootecnistas de Colombia. Colombia. Recuperado de: http://www. comvezcol.org/noticias/detalle.php?StrNot=90

Crudeli, G. (2010). Conferencia sobre Fisiología Reproductiva del Búfalo. Seminario Internacional sobre Búfalo de agua en el Trópico. San José, Costa Rica: Universidad de Costa Rica.

Erales, J., Ortega, A., Rodríguez, J., \& Segura, J. (2008). Estado y alteraciones del aparato reproductor de vacas sacrificadas en el rastro de Umán, Yucatán. Universidad y Ciencia Trópico Húmedo, 24(2),111-116

Hafez, E.S.E. (1996). Reproducción e Inseminación Artificial en Animales. México: Editorial Interamericana.

Holmann, F. Rivas, L. Pérez, E. Castro, C. Schuetz, P., \& Rodríguez, J. 2007. La Cadena de Carne Bovina en Costa Rica: Identificación de Temas Críticos para Impulsar su Modernización, Eficiencia y Competitividad. Cali, Colombia. Recuperado de: http://www. corfoga.org/images/public/documentos/pdf/ cadena_carne_bovina_CR.pdf

Khan, M., \& Khan, A. (1989). Frecuency of pregnant animals slaughtered at Faisalabad abattoir. Journal. Islamic Academic. Science, 3,82-82.

Méndez, J. (2008). Manual de recomendaciones para el manejo sostenible de la ganadería bovina de carne en la región Chorotega. MAG, Costa Rica. Recuperado de: http:// www.mag.go.cr/bibliotecavirtual/a00148.pdf

Ministerio de Economía, Industria y Comercio (MEIC). (2009). Márgenes de comercialización de la carne bovina. San José, Costa Rica. Recuperado de: http://corfoga. org/2012/wp-content/uploads/2013/07/Margenesde-Comercializaci\%C3\%B3n-de-la-Carne-BovinaMEIC-2009.pdf

Morales, S. (1985). Determinación del porcentaje de preñez en hembras sacrificadas en Coope Montecillos, Alajuela (Tesis de Bachillerato). ITCR, San Carlos, Alajuela.

Muller, E. (1982). Aspectos Reproductivos de Hembras Bovinas sacrificadas en el Matadero Municipal de Cerrillos, Cartago, Costa Rica (Tesis de maestría). UCR-Catie, Turrialba.

Ndi, C. Tambi, N., \& Agharid, M. (1993). Reducing calf wastage from the slaughtering pregnant cows in Cameroon. Revista Mundial de Zootecnia, FAO. Recuperado de: http://www.fao.org/ag/aga/agap/frg/feedback/war/ v1650b/v1650b00.htm\#Contents

Oses, B. (2002). Situación general de la producción de carne bovina. Servicio de Información de Mercados, CNP, Costa Rica. Recuperado de: http://www.mercanet.cnp.go.cr/ SIM/Estadisticas_Pecuarias/Anuario_2001.pdf

Pérez, E. (Sin año). El hato bovino nacional: Situación actual y sus proyecciones. CORFOGA, Costa Rica. Recuperado de: http://www.corfoga.org/pdf/revvol04/El_Hato_ Bovino_nacional.pdf

Pérez, E. (2010). La crisis de precios del ganado en Costa Rica. Alajuela, Costa Rica. Revista ECAG Informa No. 53.

Quintana, C. (1996). Elementos de inferencia estadística. San José. Costa Rica: Editorial de la Universidad de Costa Rica. 
Shekarchian, S., Shekarchian, S., Motaghi, M., \& Jazayeri, A. (2012). Incidence of fetal wastage in cattle slaughtered at the Shahrekord Industrial Slaughter House, Shahrekord, Irán. Research Journal of Biological Sciences, 7(4), 188-191.

Simenew, K., Bekana, M., Fikre, L., Tilahum, Z., \& Wondú, N. (2011). Major Gross Reproductive Tract Abnormalities in Female Cattle Slaughtered at Sululta Slaughter House in Ethiopia. Global Veterinaria, 6(6), 506-513.
Ugalde, G. (1996). Administración de Empresas Agropecuarias. San José, Costa Rica: EUNED.

Vázquez, M., Molina, A., Mazón, M., Brito, J., Soto, R., \& Martínez, R. (1993). Determinación del estado reproductivo del ganado bovino sacrificado en tres rastros municipales del estado de Guerrero. Revista Veterinaria de México 24 (2). Recuperado de: http://new.medigraphic.com/cgibin/resumen.cgi?IDREVISTA=39\&IDARTICULO=14344\&I DPUBLICACION=1307 